\title{
Levels of interluekin-6 and its correlation with maternal outcome in patients of gestational diabetes mellitus
}

\author{
Ujjwala Anand*, Manish Kumar, Rashmi B.
}

Department of Obstetrics and Gynecology, ESIC Model Hospital, Basaidarapur, New Delhi, India

Received: 27 January 2021

Accepted: 04 March 2021

\author{
*Correspondence: \\ Dr. Ujjwala Anand, \\ E-mail: ujjwala.anand@gmail.com
}

Copyright: (C) the author(s), publisher and licensee Medip Academy. This is an open-access article distributed under the terms of the Creative Commons Attribution Non-Commercial License, which permits unrestricted non-commercial use, distribution, and reproduction in any medium, provided the original work is properly cited.

\section{ABSTRACT}

Background: Gestational diabetes mellitus (GDM) is a glucose tolerance disorder that occurs or is diagnosed for the first time during pregnancy. Objective was to see the levels of interluekin- 6 and its correlation with maternal outcome in patients of gestational diabetes mellitus.

Methods: The source of data for the study is the antenatal patients attending the Outpatient Department or admitted in "Upper India Sugar Exchange Maternity Hospital", Department of Obstetrics and Gynaecology, G.S.V.M. Medical College, Kanpur. This study was a prospective study done on 120 patients.

Results: Most patients in the study group belonged to the age group 20-24 years followed by age group 25-29 years. Level of interleukin-6 was significantly raised in gestational diabetes mellitus patients compared to non-gestational diabetes mellitus. $13.19 \%$ patients of GDM had PROM, which was only 6.5\% in non GDM patients. $25.79 \%$ patients of GDM had preterm, which was only $12.8 \%$ in non GDM patients. 5.16\% patients of GDM had polyhydramnios, which was only $2.64 \%$ in non GDM patients. $17.20 \%$ patients of GDM had IUGR, which was only $10.31 \%$ in non GDM patients.

Conclusions: Gestational diabetes is associated with various maternal complications i.e. preeclampsia, preterm, PROM, IUGR, polyhydramnios, increased operative morbidity, development of type $2 \mathrm{DM}$.

Keywords: Interluekin-6, IUGR, Polyhydramnios, Preeclampsia, Preterm, PROM

\section{INTRODUCTION}

The term gestational diabetes mellitus was coined by O'Sullivan in $1961 .{ }^{1}$ The definition applies whether only diet modification or insulin is used for treatment and whether or not the condition persists after pregnancy. Most often this abnormal glucose tolerance returns to normal values postpartum. Thus, reclassification of maternal glycaemic status should be performed at least 6 weeks after delivery.

GDM is a public health problem that currently affects a large part of female population and has short and long term consequences for both mother as well as neonate. Prevalence of GDM varies widely. Depending on the population studied and the diagnostic test employed, prevalence may range from 2.4 to 21 per cent in all pregnancies. In a random survey performed in various cities in India, an overall GDM prevalence of $16.55 \%$ was observed. ${ }^{2}$ The studies conducted on the true prevalence of GDM in India are still very few and mostly limited to South Indian states.

IL-6 is one of several proinflammatory cytokines in the pathophysiology of insulin resistance in diabetes. In GDM, there is insulin resistance and IL-6 secretion may aggravate insulin resistance in pregnancy and participate pathogenesis of GDM. IL-6 is widely expressed in the female reproductive tract and gestational tissues, and exerts regulatory functions in embryo implantation and placental development, as well as the immune adaptations required to tolerate pregnancy.

GDM is associated with a number of risk factors. Individuals who are at high risk for GDM include: older women, women with previous history of babies with 
macrosomia, women from high-risk ethnic groups and women with elevated fasting or random plasma glucose levels during pregnancy. The risk factors for the development of GDM may be divided into several subgroups, which are presented below. The maternal determinants, often referred to as "traditional risk factors" are: higher maternal age, increased body weight, higher parity, previous delivery of a macrosomic infant, and family history of DM.

GDM causes a number of maternal and foetal short and long term complications. Maternal complications include preeclampsia, preterm, PROM, polyhydramnios, IUGR, maternal morbidity due to increased operative incidence, and long term risk of developing Type 2 DM and recurrence of GDM in subsequent pregnancies.

Most cases of GDM are managed by medical nutrition therapy and only a few require insulin support. Because of such wide range of short and long term adverse consequences, it is very important to diagnose GDM at an early stage and attain adequate maternal glycaemic control which obviates most of the associated complications.

Hence an attempt was made to know the levels of interluekin- 6 and its correlation with maternal outcome in patients of gestational diabetes mellitus.

\section{METHODS}

The source of data for the study was the antenatal patients attending the Outpatient Department or admitted in "Upper India Sugar Exchange Maternity Hospital", Department of Obstetrics and Gynaecology, G.S.V.M. Medical College, Kanpur. This study was a prospective study done on 120 patients. Subjects of the present study were divided into two groups

\section{GDM group}

The GDM group included 60 patients with 2 hour OGTT value more than $140 \mathrm{mg} / \mathrm{dl}$ (using DIPSI criteria).

\section{Non GDM group}

The non GDM group included 60 patients with 2 hour OGTT value below $140 \mathrm{mg} / \mathrm{dl}$.

\section{Inclusion criteria}

All pregnant women attending antenatal clinic, primigravida/multigravida.

\section{Exclusion criteria}

ANC cases where IL-6 is already raised preterm labour pains, premature rupture of membrane, preeclamptic toxaemia, History of intake of drugs that affect glucose metabolism like corticosteroids. Patients with Autoimmune disorders

\section{Method of case study}

All the pregnant women attending antenatal clinic who were selected for the present study, belonging to both GDM and non GDM groups, were studied in detail with regards to the clinical history, general examination, local examination, basic investigations and special investigations.

\section{History taking}

Name, address and contact number, age, gravida, parity, occupation, education and per capita income.

\section{Menstrual history}

LMP, EDD by LMP, previous menstrual cycle were noted.

\section{Obstetric history}

History of present pregnancy: Duration of amenorrhoea, symptoms regarding various trimesters and specific symptoms (on followed patients) e.g. history of headache, epigastric pain or right upper quadrant pain, blurring of vision or decreased perception of fetal movement, etc.

History of past pregnancy: History of GDM in past pregnancy, history of unexplained pregnancy loss in past, history of big sized baby in past pregnancy, history of congenital fetal anomaly in past pregnancy and history of polyhydramnios/preeclampsia/preterm/PROM in past pregnancy.

\section{Past history}

Any history of hypertension, diabetes mellitus, chronic renal disease, cardiovascular disease or any significant medical disease.

\section{Family history}

Any history of hypertension, diabetes mellitus, cardiovascular disease or history of GDM in family.

\section{Examination}

General physical examination: Nutritional status, pallor, oedema, blood pressure, weight and height.

Systemic examination: CVS, Chest, CNS and GIT examination.

Obstetrical examination: Fundal height in weeks, symphysio-fundal height in centimetres, abdominal girth, amount of liquor, lie, presentation and fetal heart rate. 
Per speculum and per vaginal examination was done as and when required.

All patients included in the study were followed till delivery and frequent glucose monitoring was done. They were managed by giving either medical nutrition therapy alone or with insulin as required. Special emphasis was given to note the presence of risk factors like age, parity, BMI, family history of diabetes, past obstetric history of GDM or macrosomic baby, socioeconomic status, etc.

Maternal complications were noted in all these patients in the form of: preeclampsia, preterm, prom, iugr, polyhydramnios.

All the patients who were diagnosed as GDM were counseled to come at 6 and 12 weeks postpartum for repeat OGTT to identify those who reverted back to normoglycaemic status.

\section{Investigations}

Following investigations were done in each case in GDM as well as non GDM group: 1) Blood group and $\mathrm{Rh}$ typing. 2) Haemoglobin estimation and complete haemogram including peripheral blood smear. 3) Urine routine and microscopic examination: Quantitative estimation of urine was done whenever it was indicated. 4) Serological markers of HIV, HBsAg. 5) VDRL test. 6) Blood sugar level: fasting and post prandial. 7) Ultrasonography for fetal well-being, AFI, EFW.

Special investigation: 2 hour- 75 gm oral glucose tolerance test was performed to all the patient on first visit and repeated 24-28 weeks of pregnancy and they was divided into study and control as per the test findings (based on DIPSI guidelines) as follows on- test positive = study group, test negative $=$ control group. OGTT was repeated in the positive patients 2 month post-partum to differentiate overt diabetes and GDM cases.

Specific investigation: IL-6 levels was measured by ELISA at 24 to 28 weeks of pregnancy.

\section{Statistical analysis}

The data was analysed using SPSS 16 software and binary logistic regression analysis to study the statistical significance of associated between various parameters. The odd's ratio and $95 \%$ confidence value was calculated to assess the significance of the study findings for general population.

\section{RESULTS}

$45.02 \%$ of the patients were in the age group 20-24 years and $32.86 \%$ patients were 25-29 years. Thus most patients in the study group belong to the age group 20-24 years followed by age group 25-29 years. As seen in the Figure 1, 56.63\% patients in GDM group were in the age group of $25-29$ years and $21.77 \%$ patients were between 20-24 years of age. Thus, most of the patients in the GDM group belong to the age group of 25-29 years.

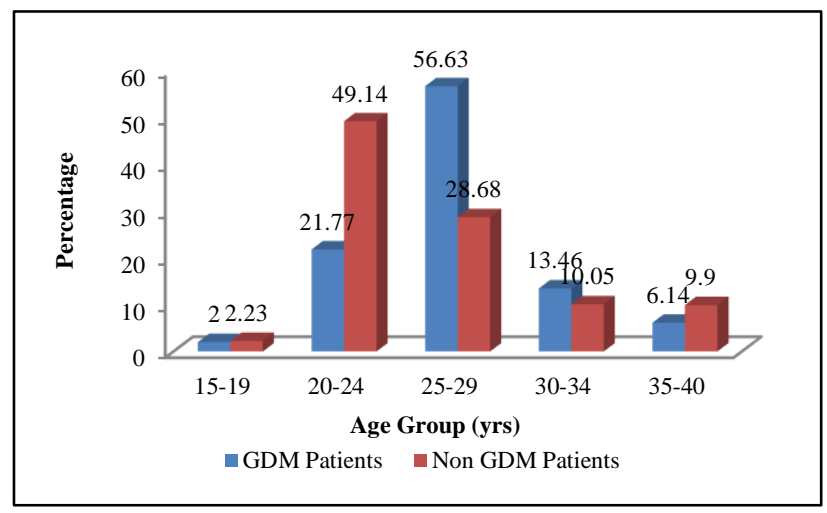

Figure 1: Comparison of age groups in GDM and non GDM population.

In the non GDM group, $49.14 \%$ patients were in the age group of $20-24$ years and $28.68 \%$ patients were between 25-29 years of age. Thus, most of the patients in the non GDM group belong to the age group of 20-24 years (Figure 1).

Most patients in the study belong to lower socioeconomic status. In the GDM group, $83.4 \%$ patients belong to lower socioeconomic status followed by $11.7 \%$ in middle status. In the non-GDM group, $84.8 \%$ patients belong to lower socioeconomic status followed by $12.3 \%$ in middle status. There was no statistically significant difference in socioeconomic status amongst GDM and non-GDM groups.

Table 1: Level of interleukin-6 in study group.

\begin{tabular}{|lll|}
\hline & $>86(\mathrm{pg} / \mathrm{ml})$ & $<86(\mathrm{pg} / \mathrm{ml})$ \\
\hline GDM & $98 \%$ & $2 \%$ \\
\hline Non GDM & $5 \%$ & $95 \%$ \\
\hline
\end{tabular}

As from the Table 1 observations level of interleukin-6 was significantly raised in gestational diabetes mellitus patients compared to non-gestational diabetes mellitus.

Table 2: Preeclampsia as a maternal complication of GDM.

\begin{tabular}{|llllll|}
\hline & & \multicolumn{4}{c|}{ Preeclampsia } \\
\cline { 3 - 6 } & & & No & Yes & Total \\
\hline \multirow{3}{*}{ Groups } & Count & 44 & 16 & 60 \\
\cline { 3 - 6 } & GDM & $\%$ & $83.8 \%$ & $16.2 \%$ & $100 \%$ \\
\cline { 3 - 6 } & \multirow{2}{*}{ GDM } & Count & 55 & 5 & 60 \\
\cline { 3 - 6 } Total & $\%$ & $77.1 \%$ & $22.9 \%$ & $100 \%$ \\
\hline & & Count & 99 & 21 & 120 \\
\cline { 3 - 6 } & & $\%$ & $82.8 \%$ & $17.2 \%$ & $100.0 \%$ \\
\hline
\end{tabular}

$\mathrm{P}<0.002$ (significant); odd's ratio $=1.539 ; 95 \%$ confidence interval $=1.167-2.030$ 
$22.9 \%$ patients of GDM had preeclampsia, which was only $16.2 \%$ in non GDM patients. There was a statistically significant difference in incidence of preeclampsia between GDM and non GDM group $(p<0.002)$. The odd's ratio was 1.539 which means that in the present study, there was approximately 1.5 times chance of preeclampsia in GDM patients than non GDM ones. The lower limit of $95 \%$ confidence interval was 1.167 which means that in general population, there was at least 1.2 times chance of preeclampsia in GDM patients when compared to non GDM ones (Table 2).

Table 3: PROM as a maternal complication of GDM.

\begin{tabular}{|llllll|}
\hline \multirow{3}{*}{ Groups } & & \multicolumn{3}{c|}{ PROM } & Total \\
& Non & No & Yes & \\
\cline { 3 - 6 } & GDM & Count & 53 & 7 & 60 \\
\cline { 2 - 6 } & \multirow{2}{*}{ GDM } & Count & 57 & 3 & 60 \\
\cline { 3 - 6 } Total & & Count & 110 & $10.5 \%$ & $100.0 \%$ \\
\hline & & $\%$ & $92.5 \%$ & $7.5 \%$ & $100.0 \%$ \\
\hline
\end{tabular}

$\mathrm{P}<0.001$ (significant); odd's ratio $=2.185 ; 95 \%$ confidence interval $=1.527-3.126$

$13.19 \%$ patients of GDM had PROM, which was only $6.5 \%$ in non GDM patients. There was a statistically significant difference in incidence of PROM between GDM and non GDM group ( $\mathrm{p}<0.001)$. The odd's ratio is 2.185 which means that in the present study, there is approximately 2 times chance of PROM in GDM patients than non GDM ones. The lower limit of $95 \%$ confidence interval is 1.527 which means that in general population, there is at least 1.5 times chance of PROM in GDM patients when compared to non GDM ones (Table 3 ).

Table 4: Preterm as a maternal complication of GDM.

\begin{tabular}{|c|c|c|c|c|c|}
\hline & & & \multicolumn{2}{|c|}{ Preterm } & \multirow{2}{*}{ Total } \\
\hline & & & No & Yes & \\
\hline \multirow{4}{*}{ Groups } & \multirow{2}{*}{$\begin{array}{l}\text { Non } \\
\text { GDM }\end{array}$} & Count & 57 & 3 & 60 \\
\hline & & $\%$ & $87.20 \%$ & $12.8 \%$ & $100.0 \%$ \\
\hline & \multirow{2}{*}{ GDM } & Count & 55 & 15 & 60 \\
\hline & & $\%$ & $74.21 \%$ & $25.79 \%$ & $100.0 \%$ \\
\hline \multirow{2}{*}{ Total } & & Count & 102 & 18 & 120 \\
\hline & & $\%$ & $85.25 \%$ & $14.75 \%$ & $100.0 \%$ \\
\hline
\end{tabular}

$\mathrm{P}<0.001$ (significant); odd's ratio $=2.369 ; 95 \%$ confidence interval $=1.801-3.115$

$25.79 \%$ patients of GDM had preterm, which was only $12.8 \%$ in non GDM patients. There was a statistically significant difference in incidence of preterm between GDM and non GDM group $(\mathrm{p}<0.001)$. The odd's ratio was 2.369 which means that in the present study, there is approximately 2.4 times chance of preterm in GDM patients than non GDM ones. The lower limit of $95 \%$ confidence interval was 1.801 which means that in general population, there was at least 1.8 times chance of preterm in GDM patients when compared to non GDM ones (Table 4)

Table 5: Polyhydramnios as a maternal complication of GDM.

\begin{tabular}{|c|c|c|c|c|c|}
\hline & & & \multicolumn{2}{|c|}{ Polyhydraminos } & \multirow{2}{*}{ Total } \\
\hline & & & No & Yes & \\
\hline \multirow{4}{*}{ Groups } & Non & Count & 58 & 2 & 60 \\
\hline & GDM & $\%$ & $97.36 \%$ & $2.64 \%$ & $100.0 \%$ \\
\hline & & Count & 58 & 2 & 60 \\
\hline & GDM & $\%$ & $94.84 \%$ & $5.16 \%$ & $100.0 \%$ \\
\hline \multirow{2}{*}{ Total } & & Count & 116 & 4 & 120 \\
\hline & & $\%$ & $96.98 \%$ & $3.02 \%$ & $100.0 \%$ \\
\hline
\end{tabular}

$\mathrm{P}<0.05$ (significant); odd's ratio $=2.006 ; 95 \%$ confidence interval $=1.159-3.472$

Table 5 shows that $5.16 \%$ patients of GDM had polyhydramnios, which was only $2.64 \%$ in non GDM patients. There was a statistically significant difference in incidence of polyhydramnios between GDM and non GDM group $(\mathrm{p}<0.05)$. The odd's ratio was 2.006 which means that in the present study, there was approximately 2 times chance of polyhydramnios in GDM patients than non GDM ones. The lower limit of $95 \%$ confidence interval was 1.159 which means that in general population, there was at least 1.2 times chance of polyhydramnios in GDM patients when compared to non GDM ones.

Table 6: IUGR as a maternal complication of GDM.

\begin{tabular}{|c|c|c|c|c|c|}
\hline & & & \multicolumn{2}{|l|}{ IUGR } & \multirow{2}{*}{ Total } \\
\hline & & & No & Yes & \\
\hline \multirow{4}{*}{ Groups } & \multirow{2}{*}{$\begin{array}{l}\text { Non } \\
\text { GDM }\end{array}$} & Count & 56 & 4 & 60 \\
\hline & & $\%$ & $89.69 \%$ & $10.31 \%$ & $100.0 \%$ \\
\hline & \multirow{2}{*}{ GDM } & Count & 50 & 10 & 60 \\
\hline & & $\%$ & $82.80 \%$ & $17.20 \%$ & $100.0 \%$ \\
\hline \multirow{2}{*}{ Total } & & Count & 106 & 14 & 120 \\
\hline & & $\%$ & $88.66 \%$ & $11.34 \%$ & $100.0 \%$ \\
\hline
\end{tabular}

$\mathrm{P}<0.001$ (significant); odd's ratio $=1.807 ; 95 \%$ confidence interval $=1.321-2.473$

Table 6 shows that $17.20 \%$ patients of GDM had IUGR, which was only $10.31 \%$ in non GDM patients. There was a statistically significant difference in incidence of IUGR between GDM and non GDM group $(\mathrm{p}<0.001)$. The odd's ratio was 1.807 which means that in the present study, there was approximately 1.8 times chance of IUGR in GDM patients than non GDM ones. The lower limit of $95 \%$ confidence interval was 1.321 which means that in general population, there was at least 1.3 times chance of IUGR in GDM patients when compared to non GDM ones.

\section{DISCUSSION}

GDM is associated with an increased risk of various short- and long-term adverse outcomes. Short-term 
pregnancy complications associated with GDM include increased risk of preeclampsia, preterm, polyhydramnios, PROM. ${ }^{3,4}$ In the long-term, somewhere between $30 \%$ of women with GDM may develop type 2 diabetes and their offspring exposed to a diabetic environment in-utero are also at an increased risk for subsequent obesity and diabetes and impairment in psychomotor development. ${ }^{5-8}$

Most patients in GDM group were in age group of 25-29 years while most patients in non GDM group were in age group of 20-24 years. Similarly in the study conducted by Sreekanthan et al, 25-32 years of age was the most common age group in GDM group while 20-24 years was the most common age in the Non GDM group. ${ }^{9}$ Rajput et al conducted a study in which the highest number of participants were in the age group 21-25 years $(58.2 \%)$ and found the prevalence rate of GDM to be higher in women aged 26-30 and >30 years (11.57 and 34.8\%, respectively) compared to women aged 16-20 and 21-25 years (4.54 and $4.53 \%$, respectively). ${ }^{10}$

Level of interleukin-6 is significantly high in the patients of gestational diabetes mellitus which is crucial in the pathophysiology of insulin resistance in the patients. Most patients in the study belong to lower socioeconomic status. In the GDM group, $83.4 \%$ patients belong to lower socioeconomic status while in the non-GDM group, $84.8 \%$ patients belong to lower socioeconomic status. There is no statistically significant difference in socioeconomic status amongst GDM and non-GDM groups. This could be due to the fact that most patients in the sample were from lower SES and the sample was not uniformly distributed. Similiarly, Yang et al also did not find such an association in Chinese pregnant women while Keshavarz et al found an association between GDM with low socio-economic level in pregnant Iranian women. ${ }^{11,12}$ In a study conducted by Kanikakalyani et al, most of the patients in the study population belonged to lower socioeconomic status but while comparing between groups, most of the patients in the GDM group were of middle class while in Non GDM, they were of lower class. $^{13}$

A significant proportion of cases with GDM had preeclampsia $(22.9 \%)$, preterm $(25.79 \%)$ or PROM $(13.19 \%)$ while in Non GDM group it was present in $16.2 \%, 12.8 \%$ and $6.5 \%$ respectively. Preterm was defined as occurrence of labour pains before 37 completed weeks and these patients were given steroid coverage under tocolysis cover for adequate fetal lung maturity. PROM was defined as rupture of membranes prior to the onset of labour and they were given antibiotic coverage along with steroid coverage if preterm, followed by induction and delivery. A similar study conducted by Kalra et al revealed that the most common complications seen in GDM mothers were gestational hypertension $(36.4 \%)$ and premature rupture of membranes (PROM) was present in $18.1 \% .{ }^{14}$ Similarly the study by Gajjar et al found that most common maternal complication seen in GDM mothers was gestational hypertension (36.4\%) followed by abruptio placentae $(20 \%){ }^{15}$

Significant proportion of cases with GDM patients had polyhydramnios $(5.16 \%)$ and IUGR (17.20\%), which was only present in $2.64 \%$ and $10.31 \%$ cases in non GDM patients respectively. However in the study conducted by Kalyani et al, no IUGR cases were observed in GDM while $1.8 \%$ cases had IUGR in Non GDM group. ${ }^{13}$

\section{CONCLUSION}

Levels of interleukin-6 is significantly higher in gestational diabetes mellitus patients which is physiological important for the insulin resistance in the patients. $22.9 \%$ patients of GDM had preeclampsia, which was only $16.2 \%$ in non GDM patients. $25.79 \%$ patients of GDM had preterm, which was only $12.8 \%$ in non GDM patients. 19\% patients of GDM had PROM, which was only $6.5 \%$ in non GDM patients. There was a statistically significant difference in incidence of PROM between GDM and non GDM group ( $\mathrm{p}<0.001)$. 5.16\% patients of GDM had polyhydramnios, which was only $2.64 \%$ in non GDM patients. $17.20 \%$ patients of GDM had IUGR, which was only $10.31 \%$ in non GDM patients.

\section{ACKNOWLEDGMENTS}

Authors would like to thank all the patients who took part in the study.

\section{Funding: No funding sources \\ Conflict of interest: None declared}

Ethical approval: The study was approved by the Institutional Ethics Committee

\section{REFERENCES}

1. O'Sullivan JB, Mahan CM. Criteria for the oral glucose tolerance test in pregnancy. Diabetes.1964;13:278-85.

2. Seshiah V, Balaji V, Balaji MS, Sanjeevi CB, Green A. Gestational diabetes mellitus in India. J Assoc Phys India. 2004;52:707-11.

3. Hod M. Clinical and experimental advances in diabetic embryopathy. Diabetic Rev Int. 1997;6:10- 4

4. Adams KM, Hongzhe L, Nelson RL, Ogbum PL, Danilenko-Dixon DR. Sequelae of unrecognized gestational diabetes. Am J Obstet Gynecol. 1998;178:132.

5. Damm P, Kuhl K, Bertelsen A, Molsted-Pedersen L. Predictive factors for the development of diabetes in women with previous gestational diabetes mellitus. Ani J Obstet Gynecol. 1992;167:607-16

6. Kaufmann RC, Schleyhahn FT, Hufhan DG, Amankwah KS. Gestational diabetes diagnostic cnteria: long term materna1 follow-up. Am J Obstel Gynecol. 1995;172:62.

7. Kjos SL, Peters RK, Xiang A, Thomas D, Schaefer $\mathrm{U}$, Buchanan TA. Contraception and the risk of type 
2 diabetes mellitus in Latina women with prior gestational diabetes mellitus. JAMA. 1998;280:5338.

8. Silverman BL, Metzger BE, Cho NM, Loeb CA. Impaired glucose tolerance in adolescent offspring of diabetic mothers- relationship to fetal hyperinsulinism. Diabetes Care. 1995;18:611.

9. K Sreekanthan, A Belicita, k Rajendran, Anil Vijayakumar, Prevalence of Gestational Diabetes Mellitus in a Medical College in South India: A Pilot Study, Indian Journal of Clinical Practice, 2014, 25,(4), 342-347.

10. Rajput R, Yadav Y, Nanda S, Rajput M. Prevalence of gestational diabetes mellitus and associated risk factors at a tertiary care hospital in Haryana. Indian $\mathbf{J}$ Med Res. 2013;137(4):728-33.

11. Yang X, Hsu-Hage B, Zhang H, Yu L, Dong L, Li J, et al. Gestational diabetes mellitus in women of single gravidity in Tianjin City, China. Diabetes Care. 2002;25:847-51.

12. Keshavarz M, Cheung NW, Babaee GR, Moghadam HK, Ajami ME, Shariati M. Gestational diabetes in
Iran: incidence, risk factors and pregnancy outcomes. Diabetes Res Clin Pract. 2005;69:279-86

13. Kalyani KR, Jajoo S, Hariharan C, Samal S. Prevalence of gestational diabetes mellitus, its associated risk factors and pregnancy outcomes at a rural setup in Central India. Int J Reprod Contracept Obstet Gynecol. 2014;3(1):219-24

14. Kalra P, Kachhwaha CP, Singh HV. Prevalence of gestational diabetes mellitus and its outcome in western Rajasthan. Indian $\mathbf{J}$ Endocrinol Metab. 2013;17(4):677-80.

15. Gajjar F, Maitra K. Intrapartum and perinatal outcomes in women with gestational diabetes and mild gestational hyperglycemia. J Obstet Gynaecol India. 2005;55:135-7.

Cite this article as: Anand U, Kumar M, Rashmi B. Levels of interluekin- 6 and its correlation with maternal outcome in patients of gestational diabetes mellitus. Int J Reprod Contracept Obstet Gynecol 2021;10:1545-50. 